\title{
Non-spherical polymersomes driven by directional aromatic interactions
}

\author{
Mingyan Luo, Yunjun Luo and Xiaoyu Li
}

Polymersomes, nanoscopic polymer vesicles self-assembled from synthetic block copolymers, are ideal candidates for drug delivery and synthetic biology, due to their characteristic of high stability, chemical versatility and surface modifiability. Besides the dimensions, researchers have demonstrated that the shape of polymersomes also plays a crucial role in their performance [1]. However, the formation of polymersomes is largely driven by the hydrophobic effect [2], which is non-directional, and thus the resultant polymersomes are mostly spherical. Anisotropic polymersomes or vesicular micelles have been reported by van Hest, Wilson and co-workers, on the conversion of spherical polymersomes into nonspherical shapes [3] by manipulating the balance between bending energy and osmotic energy, resulting prolates, discs, stomatocytes and even tubes. Li et al. reported that, by introducing a liquid crystalline block into a triblock terpolymer, polymersomes could be further transformed into polygonal or sharp-angled loop-like micelles [4]. However, barely any studies have been reported on the size control of non-spherical polymersomes.

Recently, a supramolecular strategy [5] was proposed to prepare size-controllable non-spherical polymersomes by perylene-containing diblock terpolymer (Fig. 1a), via the directional interactions between hydrophobic aromatic perylene. The perylene aromatic interactions could induce the self-assembly of amphiphilic polymers in a solvent mixture of tetrahydrofuran (THF) and water through a solvent switch method (Fig. 2a). When the plasticizing solvent THF was fully evaporated, five distinct aggregate morphologies with controllable sizes, namely: small ellipsoidal micelles (SE), medium ellipsoidal polymersomes (ME), large ellipsoidal polymersomes (LE), giant ellipsoidal polymersomes (GE), and tubular polymersomes (TU) (Fig. 1b) were identified. Since water led to more pronounced aggregation of perylenes on the

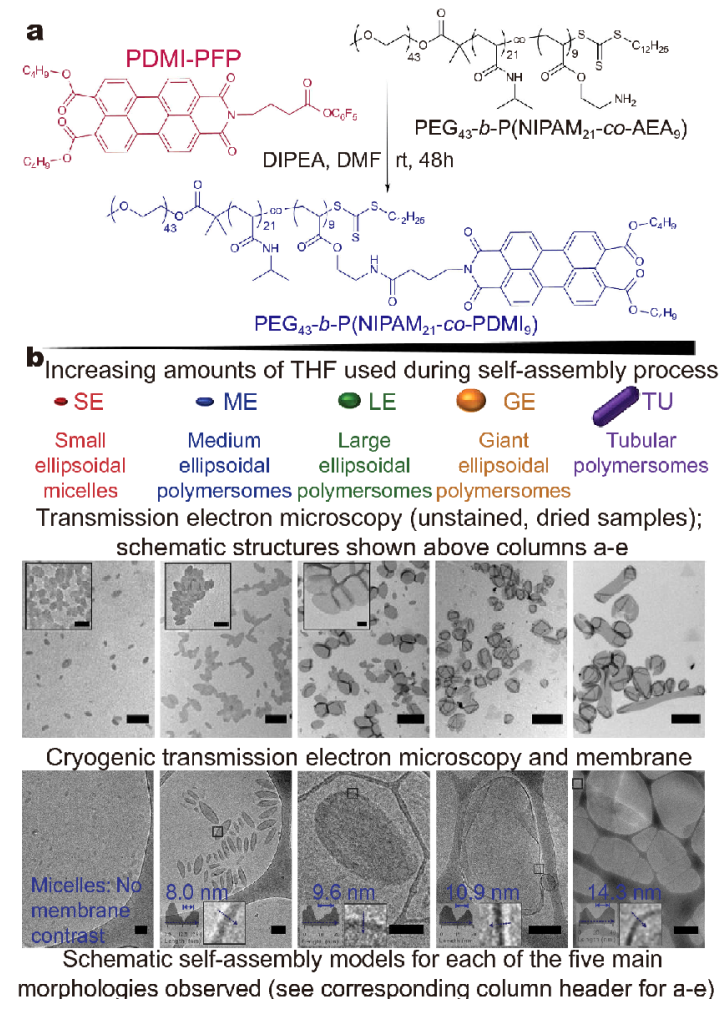

Figure 1 (a) Chemical structure of the copolymer $\mathrm{PEG}_{43}-b-\mathrm{P}\left(\mathrm{NIPAM}_{21}-\right.$ co-PDMI 9 ) used in this study. (b) TEM images and proposed self-assembly pathways for the different morphologies observed. The insets are higher magnification TEM images. Reproduced with permission from Ref. [5]. Copyright 2017 Nature Publishing Group.

diblock terpolymer, the aromatic ( $\pi-\pi$ stacking) interactions between the perylene units on the polymer are strongest in SE and gradually weakened in the larger aggregates (Fig. 1b). The combination of directional and hydrophobic aromatic interactions between the perylene units led to different spatial arrangement of perylene units, different membrane thicknesses and sizes of poly- 

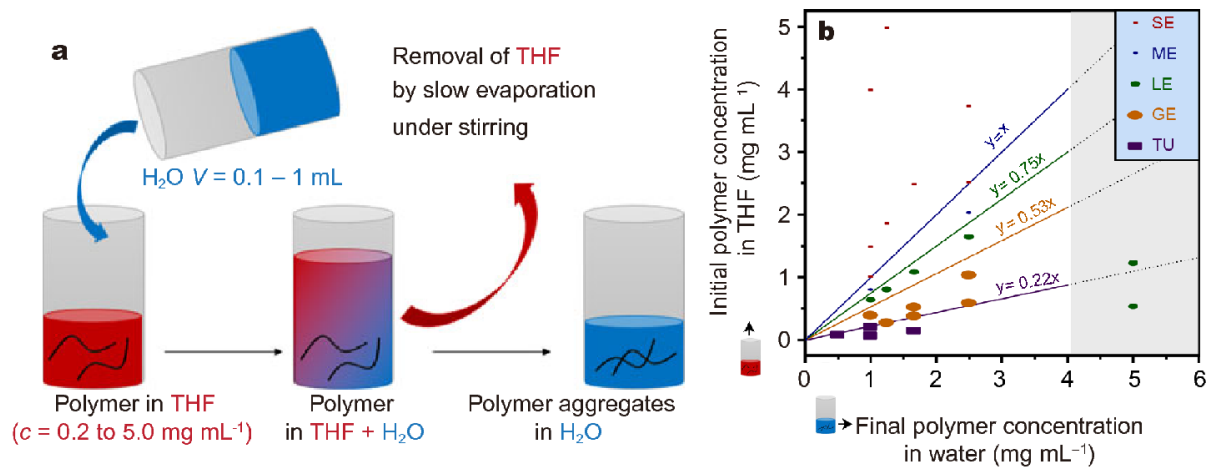

Figure 2 (a) Schematic representation depicting the solvent-switch method used to induce self-assembly of $\mathrm{PEG}_{43}-b-\mathrm{P}\left(\mathrm{NIPAM} \mathrm{M}_{21}-c o-\mathrm{PDMI} \mathrm{I}_{9}\right)$. (b) Phase diagram for the self-assembly of $\mathrm{PEG}_{43}-b-\mathrm{P}\left(\mathrm{NIPAM}_{21}-c o-\mathrm{PDMI}_{9}\right)$ using different THF:water solvent systems. Reproduced with permission from Ref. [5]. Copyright 2017 Nature Publishing Group.
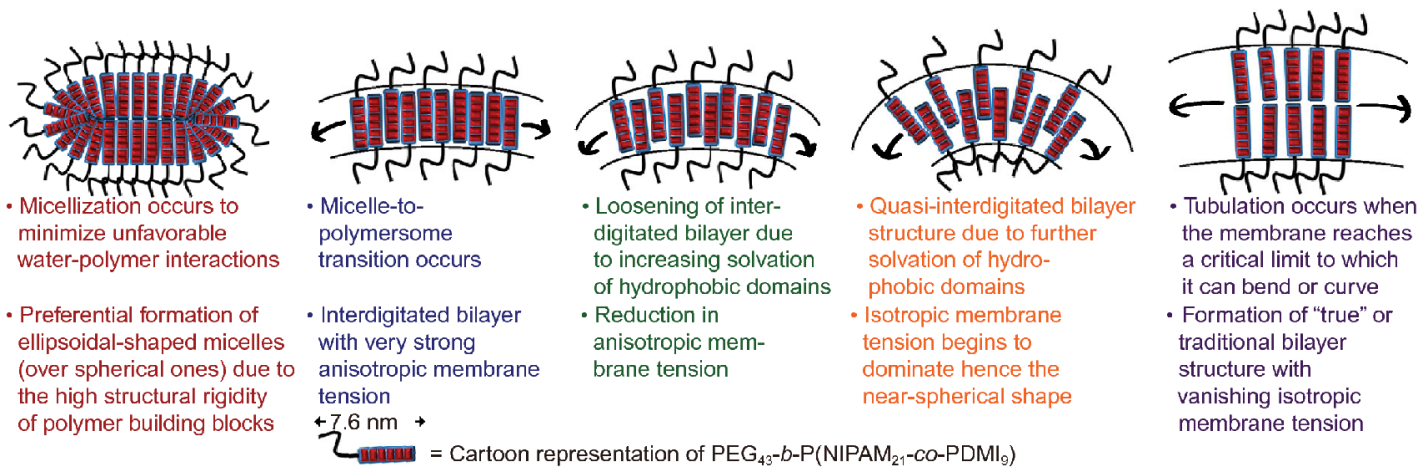

Figure 3 Schematic representations of the micelle/polymersome structures formed. Reproduced with permission from Ref. [5]. Copyright 2017 Nature Publishing Group.

mersomes (Fig. 3). A self-assembly phase diagram was drawn about the transformation of five structures, in which linear relation distinguishing five shapes between the concentrations of diblock terpolymer solution in THF and water was established (Fig. 2b).

In summary, Wong et al. demonstrated that aromatic interactions can direct hydrophobic interactions between aromatic-bearing polymers to generate non-spherical polymersomes, which can be tuned into various shapes and sizes simply by controlling the extent of solvation/ desolvation of the aromatic-bearing polymer buildings blocks, and the spatial arrangement within membranes. It can be foreseen that this facile strategy can be extended into other block copolymer systems bearing aromatic interactions [6] to prepare complex-shaped polymer particles or higher-order multicompartment structures, which can be potentially applied in drug delivery $[7,8]$.

Received 13 December 2017; accepted 14 December 2017; published online 19 December 2017

1 van der Weijden J, Paulis LE, Verdoes M, et al. The right touch: design of artificial antigen-presenting cells to stimulate the immune system. Chem Sci, 2014, 5: 3355-3367

2 Chandler D. Interfaces and the driving force of hydrophobic assembly. Nature, 2005, 437: 640-647

3 Rikken RSM, Engelkamp H, Nolte RJM, et al. Shaping polymersomes into predictable morphologies via out-of-equilibrium selfassembly. Nat Commun, 2016, 7: 12606

4 Li X, Gao Y, Xing X, et al. Polygonal micellar aggregates of a triblock terpolymer containing a liquid crystalline block. Macromolecules, 2013, 46: 7436-7442

5 Wong CK, Mason AF, Stenzel MH, et al. Formation of nonspherical polymersomes driven by hydrophobic directional aromatic perylene interactions. Nat Commun, 2017, 8: 1240

6 Sun P, Lin M, Chen G, et al. Modification of polyfluorene nanoparticles via inclusion complexation based on cyclodextrin for lectin sensing and cell imaging. Sci China Chem, 2016, 59: 16161620

7 Jia $\mathrm{H}$, Chen S, Zhuo R, et al. Polymeric prodrug for bio-controllable gene and drug co-delivery. Sci China Chem, 2016, 59: 1397-1404

8 Wang L, Zhang $\mathrm{H}$, Qin A, et al. Theranostic hyaluronic acid prodrug micelles with aggregation-induced emission characteristics for targeted drug delivery. Sci China Chem, 2016, 59: 16091615 